\title{
Strong enhancement of spontaneous emission in amorphous-silicon-nitride photonic crystal based coupled-microcavity structures
}

\author{
M. Bayindir*, S. Tanriseven, A. Aydinli, E. Ozbay \\ Department of Physics, Bilkent University, Bilkent, 06533 Ankara, Turkey \\ Received: 8 March 2001/Accepted: 17 March 2001/Published online: 23 May 2001 - @ Springer-Verlag 2001
}

\begin{abstract}
We investigated photoluminescence (PL) from one-dimensional photonic band gap structures. The photonic crystals, a Fabry-Perot (FP) resonator and a coupledmicrocavity $(\mathrm{CMC})$ structure, were fabricated by using alternating hydrogenated amorphous-silicon-nitride and hydrogenated amorphous-silicon-oxide layers. It was observed that these structures strongly modify the PL spectra from optically active amorphous-silicon-nitride thin films. Narrow-band and wide-band PL spectra were achieved in the FP microcavity and the CMC structure, respectively. The angle dependence of PL peak of the FP resonator was also investigated. We also observed that the spontaneous emission increased drastically at the coupled-cavity band edge of the CMC structure due to extremely low group velocity and long photon lifetime. The measurements agree well with the transfer-matrix method results and the prediction of the tight-binding approximation.
\end{abstract}

PACS: 42.70.Qs; 78.55.-m; 42.60.Da;78.66.Jg

Ability to control spontaneous emission is expected to have practical importance in certain commercial applications. Thus, in the past decade, photonic band gap materials were proposed for alteration (inhibition and enhancement) of the spontaneous emission from atoms [1-14].

Recently, we reported a new type of propagation mechanism in which photons move along the localized coupledcavity modes $[15,16]$. Moreover, it was observed that the group velocity tends to zero and photon lifetime increases drastically at the coupled-cavity band edges [17]. In this paper, we experimentally demonstrate the modification of spontaneous emission from the hydrogenated amorphous-siliconnitride active layers in a Fabry-Perot (FP) resonator and a coupled-microcavity (CMC) structure.

Since the density of electromagnetic modes $\varrho(\omega)$ is modified by the surrounding environments, the spontaneous emission from atoms can be controlled by placing the atoms inside

${ }^{*}$ Corresponding author.

(Fax: +90-312/266-4579, E-mail: bayindir@fen.bilkent.edu.tr) cavities. The spontaneous emission rate is directly proportional to the photon density of modes via Fermi's golden rule: $\Gamma_{\mathrm{s}} \propto \varrho(\omega) \propto 1 / v_{\mathrm{g}}$ [6]. Thus, it is expected that spontaneous emission from a CMC structure can be enhanced by a low group velocity.

Our structures were composed of alternating hydrogenated amorphous-silicon-nitride $\left(\mathrm{Si}_{3} \mathrm{~N}_{4}\right)$ and hydrogenated amorphous-silicon-oxide $\left(\mathrm{SiO}_{2}\right)$ multilayers [18]. The $\mathrm{SiO}_{2}$ and $\mathrm{Si}_{3} \mathrm{~N}_{4}$ layers were deposited on glass and silicon substrates by plasma-enhanced chemical vapour deposition (PECVD) at $250^{\circ} \mathrm{C}$. Nitrogen $\left(\mathrm{N}_{2}\right)$ balanced $2 \%$ silane $\left(\mathrm{SiH}_{4}\right)$, pure ammonia $\left(\mathrm{NH}_{3}\right)$ and nitrous oxide $\left(\mathrm{N}_{2} \mathrm{O}\right)$ were used as the silicon, nitride and oxide sources, respectively. The refractive indices and thicknesses of layers were $n_{\mathrm{SiO}_{2}}=$ $1.46, n_{\mathrm{Si}_{3} \mathrm{~N}_{4}}=1.98, d_{\mathrm{SiO}_{2}}=124.8 \mathrm{~nm}$, and $d_{\mathrm{Si}_{3} \mathrm{~N}_{4}}=92.0 \mathrm{~nm}$. The $\lambda / 2\left(d_{\text {cavity }}=184 \mathrm{~nm}\right)$ cavities were deposited with an intercavity distance $\Lambda=4.5$ pairs. The structure of the sample and experimental setup are shown in Fig. 1.

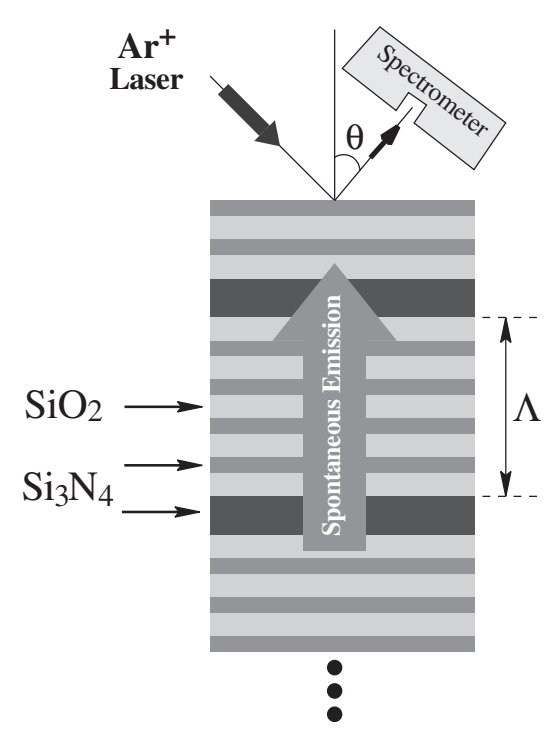

Fig. 1. Schematic of a coupled-microcavity structure and the experimental setup for measuring the photoluminescence spectra 
The room temperature photoluminescence (PL) measurements were performed using a $1 \mathrm{~m}$ double monochromator, equipped with a cooled GaAs photomultiplier tube and standard photon counting electronics, at $\theta=0^{\circ}$ with respect to the surface normal and with a spectral resolution of $2 \mathrm{~nm}$. An $\mathrm{Ar}^{+}$laser operating at $488 \mathrm{~nm}$ with $120 \mathrm{~mW}$ output power was focused with a $15 \mathrm{~cm}$ focal-length cylindrical lens on the sample. The transmission spectrum was taken by an Ocean Optics S2000 fiber spectrometer.

First, we fabricated a FP microcavity which consisted of $16 \lambda / 4$-thick $\mathrm{Si}_{3} \mathrm{~N}_{4} / \mathrm{SiO}_{2}$ pairs and a $\lambda / 2$-thick $\mathrm{Si}_{3} \mathrm{~N}_{4}$ cavity layer (see the inset in Fig. 2a). The measured transmission characteristics are displayed in Fig. 2a. We also plot the PL spectra of the FP microcavity (solid line) and a single $\mathrm{Si}_{3} \mathrm{~N}_{4}$ layer (dotted line) in Fig. 2b. In Fig. 2b, the PL spectrum of the $\mathrm{Si}_{3} \mathrm{~N}_{4}$ layer was multiplied by a factor of five. As shown in Fig. 2b, the PL spectrum was strongly modified in the presence of the FP structure. We achieved a narrow-band PL peak at wavelength $\lambda=722 \mathrm{~nm}$. Recently, similar observations have been reported by other scientists $[13,14]$.

We also measured the PL spectra at different collecting angles $\theta$ (see the inset in Fig. 3); they are plotted in Fig. 3 . We observed that the resonance wavelength was shifted towards lower wavelengths (blue-shift), and the peak intensity decreased significantly as we increased $\theta$.

Next, we fabricated a CMC structure (see Fig. 1 for the schematics of this structure) having $36 \mathrm{Si}_{3} \mathrm{~N}_{4} / \mathrm{SiO}_{2}$ pairs and four $\mathrm{Si}_{3} \mathrm{~N}_{4}$ cavity layers. Figure $4 \mathrm{a}$ shows the measured (solid line) and calculated (dotted line; using the transfer matrix method, TMM [19]) transmission characteristics of the CMC sample with four cavities. Nearly $100 \%$ transmission was achieved throughout the CMC band. We observed that (a) spontaneous emission was enhanced at the photonic band edge [6], (b) a strong enhancement of spontaneous emission was achieved for a wide

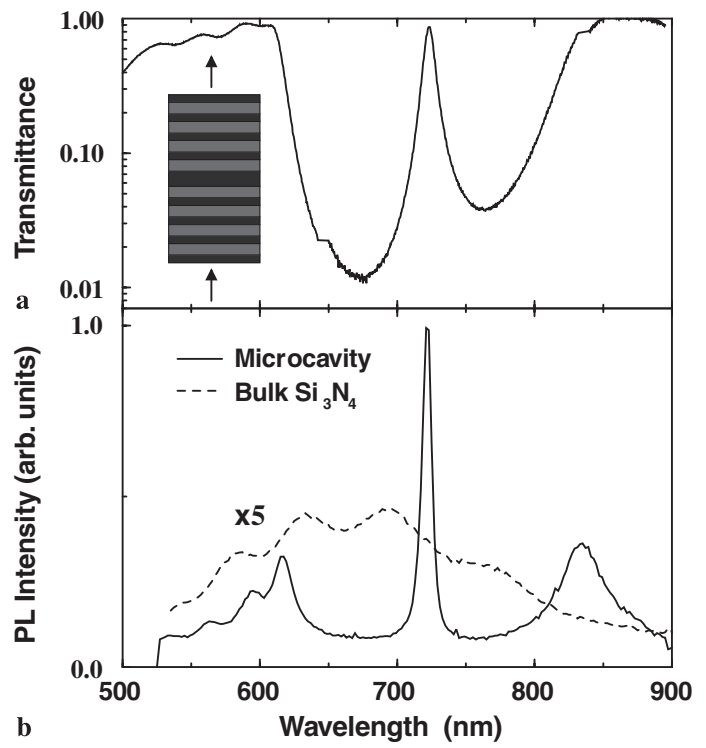

Fig. 2. a Measured transmission spectrum of a hydrogenated amorphoussilicon-nitride Fabry-Perot (FP) microcavity. Inset: Schematics of the FP microcavity structure. b Measured photoluminescence from the hydrogenated amorphous-silicon-nitride thin film (dotted line) and FP microcavity (solid line). The photoluminescence spectrum was significantly modified

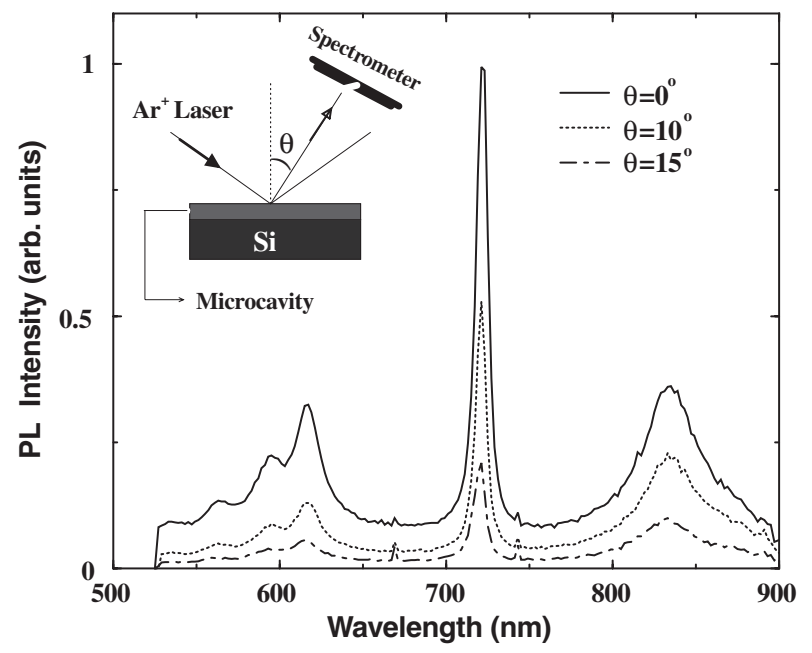

Fig. 3. Measured photoluminescence intensity as a function of wavelength for various collecting angles, $\theta$. Inset: Schematics of experimental setup for measuring the photoluminescence spectrum

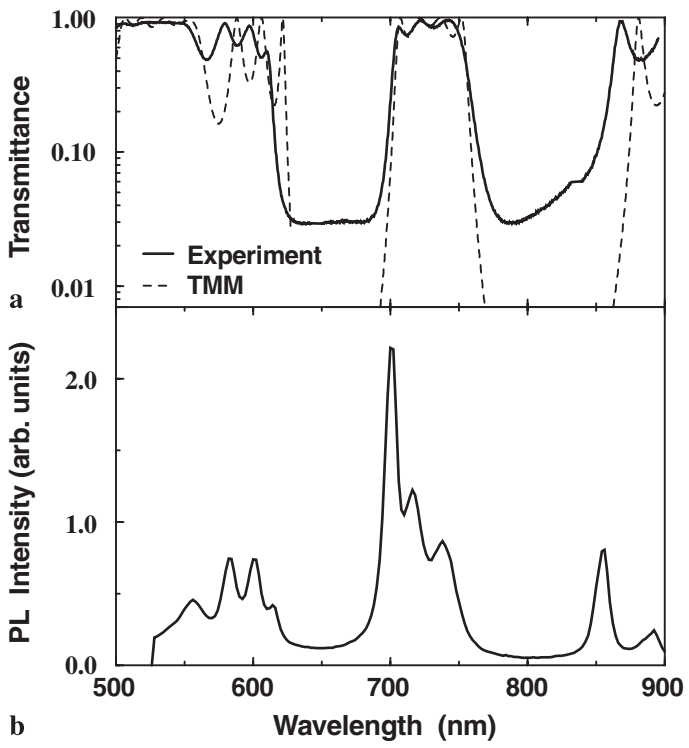

Fig. 4. a Measured (solid line) and calculated (dotted line) transmission through the $\mathrm{SiO}_{2} / \mathrm{Si}_{3} \mathrm{~N}_{4}$ coupled-microcavity (CMC) structure. Nearly $100 \%$ transmission was achieved throughout the cavity band extending from 690 to $770 \mathrm{~nm}$. b Measured photoluminescence from the CMC structure. The photoluminescence spectrum was modified, and enhanced significantly at the cavity band edge

range of wavelengths (cavity band) extending from 690 to $770 \mathrm{~nm}$, and (c) the spontaneous emission was significantly enhanced at the coupled-cavity band edge. It is important to note that the spontaneous emission displayed an oscillatory behavior near the edge of photonic band gap.

In conclusion, we investigated photoluminescence from hydrogenated amorphous-silicon-nitride Fabry-Perot microcavity and coupled-microcavity structures. We observed that the spontaneous emission spectra can be altered (inhibited or enhanced) using these structures. It was also observed that a strong enhancement of spontaneous emission can be achieved throughout the coupled-cavity band. These results open up a variety of possibilities in optoelectronic applica- 
tions, such as coupled-cavity broadband high brightness lightemitting devices.

Acknowledgements. This work was supported by NATO Grant No. SfP971970, National Science Foundation Grant No. INT-9820646, Turkish Department of Defense Grant No. KOBRA-001, and Thales JP8.04.

\section{References}

1. E. Yablonovitch: Phys. Rev. Lett. 58, 2059 (1987)

2. A.M. Vredenberg, N.E.J. Hunt, E.F. Schubert, D.C. Jacobson, J.M. Poate, G.J. Zydzik: Phys. Rev. Lett. 71, 517 (1993)

3. S. John, T. Quang: Phys. Rev. A 50, 1764 (1994)

4. R.P. Stanley, R. Houdre, U. Oesterle, M. Ilegems, C. Weisbuch: Appl. Phys. Lett. 65, 2093 (1994)

5. L. Pavesi, C. Mazzoleni, A. Tredicucci, V. Pellegrini: Appl. Phys. Lett. 67, 3280 (1995)

6. M.D. Tocci, M. Scalora, M.J. Bloemer, J.P. Dowling, C.M. Bowden: Phys. Rev. A 53, 2799 (1996)

7. L. Pavesi, G. Panzarini, L.C. Andreani: Phys. Rev. B 58, 15794 (1998)
8. A. Serpenguzel, A. Aydinli, A. Bek, M. Gure: J. Opt. Soc. Am. B 15, 2706 (1998)

9. R.K. Lee, O.J. Painter, B. D’Urso, A. Scherer, A. Yariv: Appl. Phys. Lett. 77, 1522 (1999)

10. M. Boroditsky, R. Vrijen, T.F. Krauss, R. Coccioli, R. Bhat, E. Yablonovitch: IEEE J. Lightwave Technol. 17, 2096 (1999)

11. H.A. Lopez, P.M. Fauchet: Appl. Phys. Lett. 77, 3704 (2000)

12. A.A. Dukin, N.A. Feoktistov, V.G. Golubev, A.V. Medvedev, A.B. Pevtsov, A.V. Sel'kin: Appl. Phys. Lett. 77, 3009 (2000)

13. F. Giorgis: Appl. Phys. Lett. 77, 522 (2000)

14. A. Serpenguzel, S. Tanriseven: Appl. Phys. Lett. 78, 1388 (2001)

15. M. Bayindir, B. Temelkuran, E. Ozbay: Phys. Rev. Lett. 84, 2140 (2000); M. Bayindir, B. Temelkuran, E. Ozbay: Phys. Rev. B 61, R11 855 (2000)

16. M. Bayindir, S. Tanriseven, E. Ozbay: Appl. Phys. A 72, 117 (2001)

17. M. Bayindir, E. Ozbay: Phys. Rev. B 62, R2247 (2000); M. Bayindir, E. Ozbay, B. Temelkuran, M.M. Sigalas, C.M. Soukoulis, R. Biswas, K.M. Ho: Phys. Rev. B 63, 081107(R) (2001)

18. A. Aydinli, A. Serpenguzel, D. Vardar: Solid State Commun. 98, 273 (1996)

19. M. Born, E. Wolf: Principles of Optics (Cambridge University Press, Cambridge 1998); A. Ghatak, K. Thyagarajan: Optical Electronics (Cambridge University Press, New York 1989) 